\title{
Temporary support strategies for cardiogenic shock: extracorporeal membrane oxygenation, percutaneous ventricular assist devices and surgically placed extracorporeal ventricular assist devices
}

\author{
Howard Todd Massey, Jae Hwan Choi, Elizabeth J. Maynes, Vakhtang Tchantchaleishvili \\ Division of Cardiac Surgery, Thomas Jefferson University, Philadelphia, PA, USA \\ Correspondence to: Howard Todd Massey, MD. Surgical Director, Cardiac Transplantation and Mechanical Circulatory Support Program, Division of \\ Cardiac Surgery, Thomas Jefferson University, 1025 Walnut St, Suite 607, Philadelphia, PA 19107, USA. Email: Howard.Massey@jefferson.edu.
}

\begin{abstract}
The majority of clinical pathways and paradigms utilized in the treatment and management of cardiogenic shock with temporary mechanical circulatory support (MCS) are largely based on individual physician intuition and ad hoc problem-solving. Substantial mortality gains in the acute myocardial infarction cardiogenic shock (AMI-CS) population were observed with the reported outcomes of the SHOCK trial in 1999 compared to previous populations with AMI-CS. Nonetheless even in the age of percutaneous coronary intervention (PCI) of the infarct related artery, survival rates continue to be only approximately $50 \%$. The conventional focus since the SHOCK trial has centered on revascularization strategies and the subsequent medical management of these patients post-PCI with ever diminishing returns. Perhaps we have hit the "glass ceiling" with current strategies and it is time to explore novel strategies to salvage not only the heart but more importantly the patient and potentially more of both. Going forward, researchers need to focus on developing a systematic approach to problem solving in utilizing MCS for patients with cardiogenic shock. Effective methodologies that are evidence based will help physicians in their decision-making when considering temporary MCS for patients.
\end{abstract}

Keywords: Cardiogenic shock; left ventricular assist devices (LVADs); acute myocardial infarction (AMI); percutaneous coronary intervention and extracorporeal membrane oxygenation (PCI and ECMO)

Submitted Oct 02, 2018. Accepted for publication Nov 08, 2018.

doi: 10.21037 /acs.2018.11.05

View this article at: http://dx.doi.org/10.21037/acs.2018.11.05

\section{Introduction}

Approximately $10 \%$ to $15 \%$ of patients with acute ST segment elevated myocardial infarction (STEMI) will develop cardiogenic shock which equates to approximately 60,000 cases per year (1). Cardiogenic shock in the setting of an acute STEMI generally occurs due to left ventricular (LV) dysfunction in approximately $80 \%$ of cases (2). One of the original definitions of cardiogenic shock was developed by Dr. Thomas Killip III, Chief of Cardiology at the New York Hospital during the development of Cardiac Critical Care units in the late 1960s which were specifically designed to care for patients with cardiogenic shock (3). This past year, the 50th anniversary of Cardiac Critical Care Units was celebrated in recognition of how these specialty units have substantially contributed to improved outcomes in these patients (4). The SHOCK trial, reported in the New England Fournal of Medicine in 1999, revealed a significant survival benefit at six months and at 12 months in those individuals who received percutaneous coronary intervention (PCI) of the infarct-related artery, as compared to individuals managed medically using thrombolytic therapy (5). Of note, during the index cardiogenic shock hospitalization, there was no significant survival benefit observed between the two patient groups. Subsequent to the outcomes reported in this trial, there has been an emphasis in the United States 
and internationally on improving door-to-balloon times (6). Within the United States, this has become a nationally tracked quality metric and is directly tied to Centers for Medicare and Medicaid Services reimbursement (7).

Revascularization of the infarct related artery has improved mortality outcomes from approximately $70 \%$ preceding this strategy to approximately $50 \%$. The adage "time is muscle" has become a familiar slogan across all emergency departments and cardiac catheterization labs. Approximately two decades since the SHOCK trial, the vast majority of the randomized control trials or large meta-analyses of trials have focused on revascularization strategies and the medical management of patients with AMI-CS that received PCI of the infarct related artery. One of these trials is the CULPRIT-SHOCK trial, which investigated the benefit of multi-vessel PCI including the culprit artery against the PCI of the culprit artery only (8). The SHOCK 2 trial looked at a post-PCI population who were randomized to either receive or not receive an intra-aortic balloon pump (9). Similarly, a large Cochrane database systematic review evaluated inotropic and vasodilatory agents used in the treatment of cardiogenic shock in an myocardial infarction (MI) population to assess the potential benefits of certain inotropes or vasodilator therapy (10). In this trial, the researchers specifically focused on a novel calcium sensitizer, levosimendan, compared to conventional agents administered (10). The TRIUMPH trial assessed medical treatment of patients with acute MI and specifically looked at a non-specific inhibitor of nitric oxide synthetase, tilarginine, to see if this could potentially improve patient outcomes (11). Unfortunately, none of these recently performed studies revealed any significant improvements in survival.

Even in the age of PCI of the infarct related artery, survival rates in most contemporary studies regarding AMICS continue to be only approximately $50 \%$; substantial gains in this patient population have not been observed since the reported outcomes of the SHOCK trial in 1999 (12). With the current focus on revascularization and the subsequent medical management of AMI-CS patients, perhaps we have hit the "glass ceiling" with this strategy and perhaps it is time to explore newer, novel strategies to further impact one of the highest mortality conditions that an individual can be admitted with to a hospital.

\section{Mechanical circulatory support (MCS) for acute cardiogenic shock}

When you look at the trials that have utilized MCS for cardiogenic shock, these studies are very few in number and include very few patients. Currently, there are only four randomized control trials involving temporary MCS in cardiogenic shock and none of these trials have revealed any survival benefit (13-16). Some of the trials did reveal some modest hemodynamic benefits, but this was not necessarily translated into survival benefit. Overall, out of all these studies, only approximately 200 patients in total have been involved in randomized trials utilizing MCS. Nonetheless, over the past few decades, there has been a significant increase in the utilization of MCS devices for this patient population $(17,18)$. In addition, there has been a very substantial growth in the utilization of extracorporeal membrane oxygenation (ECMO) in patients with cardiogenic shock and not necessarily with any significant improvement in survival in this ECMO population over these time periods (19).

Due to the paucity of clinical trials involving temporary MCS, most of the information in this manuscript is really based on fairly anecdotal evidence. I do believe that "what we know here is very little and what we are ignorant of is immense". The majority of clinical pathways and paradigms utilized in AMI-CS have been derived from individual practitioner intuition and ad hoc problem-solving "on the fly". Going forward, researchers need to focus on developing a systematic approach to utilizing MCS in AMICS and pool resources nationally and even internationally to guide evidence-based strategies. Performing randomized control trials in patients with AMI-CS may be neither practical nor ethical especially in regard to utilizing or not utilizing MCS. Nonetheless, scientifically sound evidencebased methodologies have to be derived using alternative study designs and statistical methods to guide physicians with appropriate decision-making in applying MCS.

\section{Cardiogenic shock management and trauma systems}

Recently, there was a call for a national initiative to develop a state level regionalized cardiogenic shock management system $(20,21)$. One of the fundamental observations made from this initiative came from looking at the national trauma system, which has been successfully applied in the US with noted improvements in patient outcomes. The system was modeled after the national trauma system as there are parallels between acute trauma care and acute cardiogenic shock care. A proposed three tier system was originally proposed with the goal of implementation at the 
regional level through statewide initiatives supported by different resources available at the participating institutions. This plan focused on regionalization of care through interinstitutional collaboration in the development of highly coordinated care delivery models based on individual institutional resources. There were multiple factors that helped to foster these inter-institutional partnerships. During this time period, there was the development of statewide initiatives in public reporting of outcomes in cardiac surgery and interventional cardiology. Overall, we found that quality reporting initiatives had consequences that were not necessarily readily apparent, especially when risk adjustment was not felt to be adequate. The potential negative implications of public quality reporting in relation to cardiogenic shock patients has been illustrated in studies concerning New York and Massachusetts, where there was a noted decrease in the utilization of PCI and coronary artery bypass grafting (CABG) in populations with acute MI cardiogenic shock $(22,23)$. The perceived institutional risk associated with AMI-CS patients helped foster interinstitutional partnerships that may not have normally developed. This did provide an opportunity to develop a regionalized network of care to help provide more effective management for this AMI-CS population. To aid with the inter-institutional transition of care of this population, we developed a cardiogenic shock critical care transport team approximately two decades ago. The development of the cardiac critical care transport team provided safe transport of these critically ill patients by a specialized team as well as it greatly facilitated the regional partnerships. Through this collaborative effort, inter-institutional care delivery models were developed for patients which helped break down institutional barriers. Through these efforts and partnerships there was the development of a highly clinically integrated regionalized network to improve care delivery models to cardiogenic shock patients.

\section{MCS systems of the past}

In the past, the majority of mechanical support systems deployed in cardiogenic shock were initially pulsatile systems. The ABIOMED BVS 5000 biventricular support system (ABIOMED BVS 5000 Inc., Danvers, Massachusetts, USA) was the first FDA approved device for this use in 1992. This device was very cumbersome, difficult to implant, required full cardiopulmonary bypass with a sternotomy, and required in most instances, full cardiopulmonary bypass support to explant the system (Figure 1). For this reason, the sternum was either left open or had to be reopened for explant. The device was also associated with a moderately high incidence of adverse complications, especially in relation to thromboembolic complications and bleeding. The threshold for implanting these systems was very high given the complexity and limitations of the technology available and the vast majority of patients that received these systems had either ongoing cardiopulmonary resuscitation (CPR) or recently administered CPR and established multisystem organ failure. Nonetheless, despite the clear obstacles to this, survival rates ranged from $29-60 \%$ in a reported series, and some of these individuals were able to go on to live full lives (Figure 2).

\section{Current MCS systems-peripheral continuous flow support devices}

Over the past two decades, since the FDA approval of the Impella ABIOMED BVS 5000, there has been a transition away from surgically placed central pulsatile pumps to more peripherally placed continuous flow devices. The peripheral devices can be broken down broadly into two groups: (I) the micro axial flow pumps which are represented by the AbioMed Impella and (II) the centrifugal para-corporeal temporary pumps. The centrifugal pumps are typically deployed as veno-arterial ECMO (VA-ECMO) systems or as percutaneous or surgically placed ventricular assist devices (VAD). One important point to consider regarding the peripherally placed pumps is that flow is proportional to cannula size. The larger the cannulas, typically the greater the flow. The most serious adverse events including limb ischemia and bleeding, are common to all the peripherally placed temporary systems. Limb ischemia and bleeding are directly proportional to the arterial cannula sizes utilized which is proportional to the flows achieved rather than the individual system brand. The larger the arterial cannula, the greater the flow but the higher the risk of bleeding or limb ischemia.

The temporary devices are mainly deployed for rapid circulatory support to reverse or prevent multi- system organ dysfunction. In many instances, they are utilized as bridges to more durable systems or cardiac transplantation. One factor to keep in mind regarding these systems, especially in relation to bridging to recovery, is the short durations of FDA approval for these devices; the Impella systems are approved for up to 6 days of use and the 


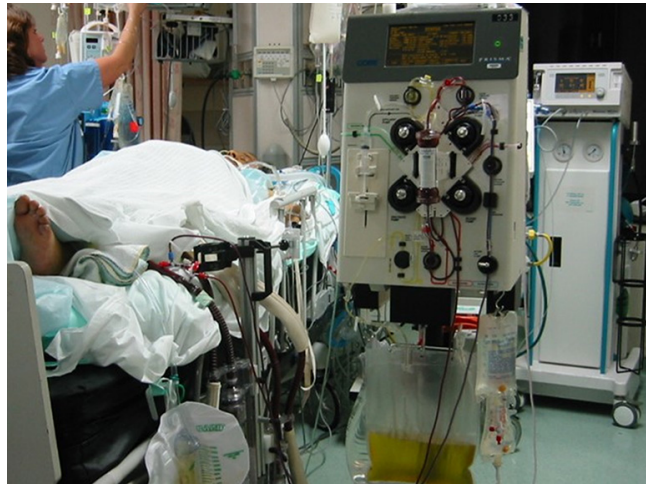

Figure 1 Patient with multisystem organ failure bridged with the Abiomed BVS 5000.

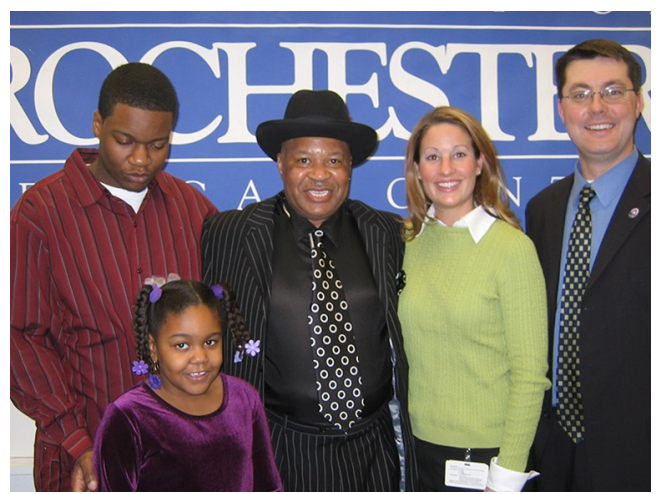

Figure 2 The same patient (Figure 1) after being successfully bridged by the Abiomed BVS 5000.

para-corporeal centrifugal pumps are only approved for 6 hours of use; this should be taken into consideration when looking at temporal patterns of native cardiac recovery. If in situations where there are favorable indicators for early native cardiac recovery or in circumstances where recovery is deemed the only option, these devices can be utilized for longer time periods with serial assessment for native cardiac recovery. Otherwise, if the duration for recovery is going to be much longer than a week to 10 days, then earlier transitioning over to a more durable system should be considered if the patient meets candidacy thresholds.

Deciding on which pump to deploy in which circumstance typically depends on the degree of flow that the clinical circumstance warrants, how rapidly one needs to restore flow, certain particular clinical circumstances, and the institutional and individual physician preference and skill. There are certain circumstances where it may be contraindicated to place a certain device. If a patient has a thrombus involving the left ventricle or the left atrium, it is best to avoid deploying devices within these cavities. In a clinical situation involving a ventricular septal defect, it is best to avoid devices that directly unload the left ventricle since this could increase right to left shunting and worsen hypoxemia. In a patient with moderate to severe aortic insufficiency, typically none of the devices will adequately restore systemic perfusion due to a reflow phenomenon. In the patient with significant aortic insufficiency, the patient will need to have the aortic valve addressed surgically or percutaneously to provide adequate tissue perfusion.

\section{VA-ECMO}

The VA-ECMO system is fairly easy to insert and requires only minimal surgical skills. Many professionals in a wide variety of disciplines, including intensivists, emergency room physicians, and interventional cardiologists have been trained to place a patient on VA-ECMO. Establishing VA-ECMO support does not necessarily require echocardiographic or fluoroscopic guidance, and for that reason it can be deployed in any location within the hospital or even outside the hospital in remote locations, such as in military and first responder situations. An important factor to consider when placing a patient on VA-ECMO is that it is not the most favorable system for myocardial recovery. It can be very effective at restoring tissue perfusion and can provide optimal flow, but unfortunately does significantly increase afterload, increases left ventricular end diastolic pressure (LVEDP), and increases myocardial oxygen demand.

VA ECMO is most rapidly deployed percutaneously in the femoral artery (15-21 Fr) and femoral vein (19-23 Fr) (Figure 3). It is important to prophylactically place either a 6 to $8 \mathrm{Fr}$ distal perfusion cannula to significantly decrease the incidence of distal limb mal-perfusion. The wires for this should be placed prior to placing the wires for the arterial cannula. Utilizing the subclavian artery cannulation through an $8-\mathrm{mm}$ vascular graft has also been done frequently. In this setting, there is a possibility for hyperperfusion of the upper extremity; however, this can be fairly easily controlled by utilizing vessel loops distal to the arterial cannulation site, which can be adjusted at the bedside using an ipsilateral radial arterial perfusion pressure. By using the subclavian artery as an outflow and the right internal jugular vein as an inflow, the patient is more readily mobilized.

VA-ECMO increases afterload and in some situations 

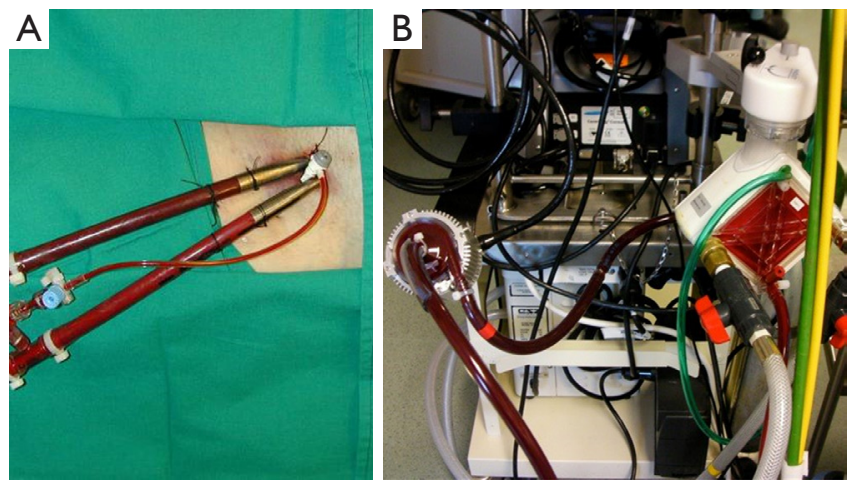

Figure 3 Veno-arterial ECMO. (A) Cannulation of femoral artery and femoral vein; (B) pump and oxygenator. ECMO, extracorporeal membrane oxygenation.

can lead to significant $L V$ distention and elevations in LVEDP requiring ventricular unloading. There are many different techniques that can be utilized to address LV distention. The initial step should be to decrease VAECMO flow while utilizing mild to moderate doses of dobutamine to increase contractility and decrease afterload to restore native heart pulsatility on the arterial line (24). If the initial method fails to restore pulsatility, the preferred and most efficacious technique that has been utilized is atrial septostomy with a trans-atrial cannula placement as an additional inflow cannula to the system. This cannula can also be placed across the mitral valve if necessary. This method to vent the $\mathrm{LV}$ is the safest method in regard to bleeding risk as access to the arterial system is through the peripheral venous system and this approach is the most effective for protecting the lungs from pressure induced microvascular damage. $\mathrm{LV}$ venting is considered in patients with severe LV dysfunction and lack of pulsatility on arterial line tracing. Echocardiogram reveals the aortic valve is not opening and there is relative distention in the presence of "smoke" within the left ventricle, signifying stasis. A Swan-Ganz catheter is always utilized on VA-ECMO patients for continuous monitoring of pulmonary artery diastolic pressure. A pulmonary artery diastolic pressure of approximately $20 \mathrm{mmHg}$ in the presence of corroborating ECHO findings should trigger methods to address LV unloading. The advantages for unloading the $L V$ outweigh the cons, as it promotes $L V$ recovery, prevents $L V$ stasis and thrombus formation, and protects the lungs from pressure related microvascular damage (Figure 4).

One of the other concerns while using femoral VAECMO is the so-called Harlequin syndrome; this is where the patient essentially develops a blue upper body (deoxygenated blood directed to the upper half of the body) and a red lower body (oxygenated blood directed to lower half of the body). The syndrome is basically derived from competition of the native heart with the ECMO system within the aortic column where the patient can have a transition zone of oxygenated and deoxygenated blood in the presence of an ejecting native heart and poorly functioning lungs. For this reason, in femoral arterial VAECMO monitoring of the right radial arterial saturations, upper extremity and head saturation probes or monitoring of cerebral near-infrared spectroscopy is essential to detect this potentially life-threatening complication that can lead to irreversible neurologic injury. Unfortunately, we are currently unable to monitor the relative amounts of hypoxemia within the aortic sinuses which could impact myocardial recovery.

Experience in mobilizing temporary MCS supported patients was gained through hybrid systems of temporary right VADs (RVADs) and implantable left VADs (LVADs) (Figure 5). A method was developed that allows for sternotomy closure and subsequent remote decannulation in these patients. It was possible to ambulate patients within the intensive care unit and take them off positive pressure ventilation; this was beneficial for rehabilitation as well as for preventing complications associated with sedation and recumbency in positive pressure ventilation. This led to improved survival and explant rates in this hybrid population. Simultaneously with this was an increased utilization of ambulation and extubation in the veno-venous ECMO group. There have been some initial positive outcomes observed in VA-ECMO patients in regard to mobilization and active rehabilitation and this will likely continue in the future. The biggest obstacle to this is the fear of inadvertent decannulation as the majority of the systems are not designed with this in mind. However, this is a technical issue and is easily solvable.

\section{ECMO support and advancements in oxygenators}

One of the major areas of technological development and improvement has been in the oxygenators and the development of biocompatible coatings. The Macquet QUADROX-D polymethyl-pentene hollow fiber membrane oxygenator with a Bioline coating improved biocompatibility, decreased flow resistance, and enhanced diffusion parameters in a fairly small sized membrane 


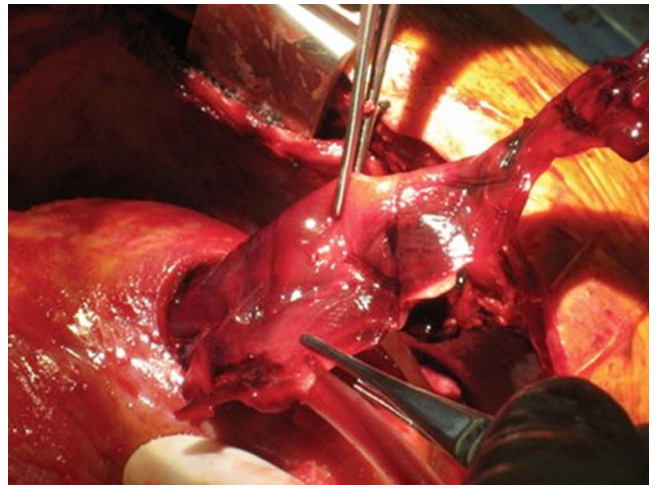

Figure 4 Thrombi being extracted from the left ventricle; thrombi formation can occur without adequate unloading.

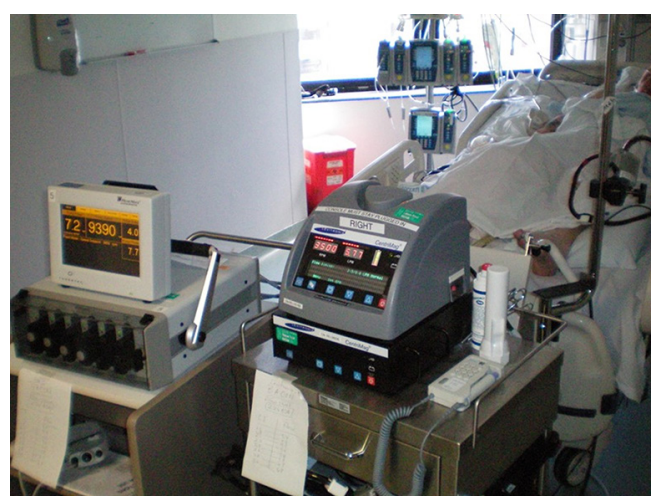

Figure 5 Patient on a biventricular support including a temporary RVAD and an implantable LVAD. RVAD, right ventricular assist device; LVAD, left ventricular assist device.

area (25). Previously utilized microporous hollow fiber polypropylene oxygenators were noted for significant blood trauma, higher resistances to flow and plasma leakage after 6 hours of utilization (Figure 6). These systems were unforgiving in attempts to forego anticoagulation.

Overall, given the improved performance of the oxygenators and pumps, anticoagulation is given during the initial placement with an activated clotting time (ACT) goal of approximately 250 seconds. At least 12 to 24 hours of time for stabilization is then allotted without anticoagulation. Anticoagulation is then typically resumed with ACT goals of approximately 150 to 180 seconds. Heparin boluses are always avoided after the initial placement. If a situation arises where anticoagulation cannot be utilized, these systems have become safe and biocompatible enough that withholding heparinization may be feasible and well tolerated with appropriate monitoring

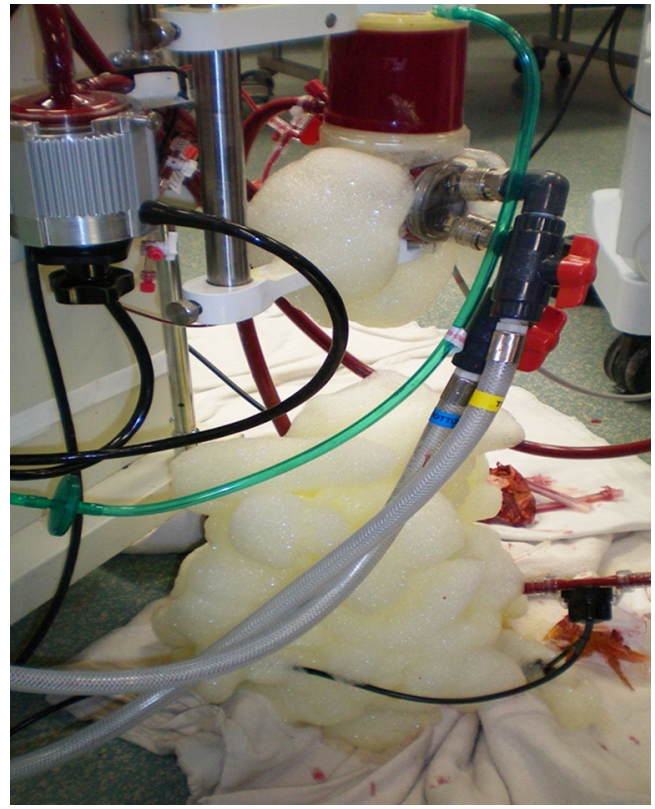

Figure 6 Plasma leakage from the microporous hollow fiber polypropylene oxygenators in ECMO. ECMO, extracorporeal membrane oxygenation.

of the system membrane oxygenator.

\section{The Maquet CardioHelp system}

The Maquet CardioHelp system was specifically designed for transportability. The CardioHelp system is ergonomically designed with an integrated pump and oxygenator specifically designed for being able to move patients within hospitals and between hospitals and at the same time provide important clinical information such as system pressures, mixed venous oxygen saturation and hematocrit utilizing the incorporated sensors within the circuit. It is a small area system that can be fully primed in less than 15 minutes.

\section{The Tandem system}

The Tandem system is a paracorporeal LVAD with a 21-Fr inflow cannula placed trans-septal into the left atrium through the femoral vein that is coupled with a 17 to $19 \mathrm{Fr}$ outflow cannula placed in the femoral artery. The Tandem system also has the capabilities for RVAD support, utilizing a unique dual lumen cannula called the Protek Duo (CardiacAssist, Pittsburgh, PA, USA) that can be placed within the right internal jugular vein and subsequently 
through the pulmonary valve. An oxygenator can also be added with the Tandem Lung. The Tandem Life centrifugal pump utilizes a hydrodynamic bearing and an infusion line integral to the pump to continuously flush through the motor housing a heparin solution in order to cool the bearings as well as to prevent deposition of fibrin within the lower pump chamber. It can provide flow of up to $4 \mathrm{~L} /$ min. It is typically placed in the cardiac catheterization lab utilizing fluoroscopic and echocardiographic guidance and it does require a special skill set to be able to transverse the septum with the inflow cannula.

\section{The ABIOMED system}

The AbioMed Impella system is a unique micro axial flow temporary LVAD system that is either percutaneously or surgically placed depending on the cannula and corresponding flow capabilities required. The pump is designed with an integrated motor and an impeller within a cannula that draws blood from the LV to the aorta while traversing the aortic valve. The system comes in multiple sizes ranging from a $13 \mathrm{Fr} / 2.5 \mathrm{LPM}, 14 \mathrm{Fr} / 3.5 \mathrm{LPM}$ up to a $22 \mathrm{Fr} / 5$ LPM model. The patients are heparinized during the initial placement of the pump, but do not require any subsequent systemic heparinization; the system is flushed with a heparinized solution through its purge system. In theory, the Impella system would produce the most favorable cardiac hemodynamics for myocardial recovery by directly unloading the left ventricle and increasing myocardial oxygen delivery while decreasing myocardial oxygen demand. The AbioMed Impella system (Danvers, MA, USA) is one of the first devices that specifically introduced a temporary LVAD system to interventional cardiologists and it has sparked a "Renaissance" within the field in relation to AMI-CS. There are problems with malposition of the pumps, hemolysis, and variable flows especially with the smaller percutaneously placed pumps.

\section{Theoretical underpinnings and operational designs}

It is clear the original intent in the development of MCS was to support patients in critical cardiogenic shock and it is the foundation upon which all subsequent developments in MCS were based upon as illustrated by the 1971 article by Dr. Michael DeBakey describing some of the first LVADs placed in three patients with post-cardiotomy cardiogenic shock. Dr. DeBakey pointed out it is essential to restore flow in a timely manner and prevent the development of multisystem organ failure. The current national algorithms for AMI-CS that are utilized today address only the $50 \%$ of patients that will survive their PCI of the infarct related artery and do not adequately address the other half of the equation (26). Even in the age of PCI, the mortality rates in varying levels of cardiogenic shock still continue to range at approximately 50\% (27). There have been major advances in the field of MCS and LVADs for patients with chronic heart failure (Figure 7). There are devices at our disposal that can be readily implanted through minimally invasive incisions that reliably pump blood for years with low adverse event profiles (28).

A lot has been learned from shock trauma and many parallels exist between cardiogenic shock and shock trauma. One of the lessons learned was the need to get people out of shock within a timely manner. In shock trauma, much emphasis is placed on getting people out of shock within one hour, the so-called "Golden Hour". If patients are not brought out of shock within that hour, they will likely die subsequent to multisystem organ failure and systemic inflammatory response, despite having their underlying problem ameliorated. In the SHOCK trial, the time to revascularization from the onset of symptoms especially in the group that underwent $\mathrm{CABG}$, the median time was approximately 20 hours and even out to 30 hours (29). This has to stimulate some questions: were these patients remaining in continuous shock for 30 hours? In these roughly $50 \%$ of people that died, at what point did they die, and at what point did they cross the "event horizon" to the point of no return, where despite restoration of flow they would continue to die from multisystem organ failure? If we could predict these outcomes on presentation, would that change what we did? In the patients that are going to improve with revascularization versus those who are not, would we address these patients differently? It is ultimately about time and not unlike with in door to balloon time where "Time is Muscle"-time was of the essence where there is certainly a time point that could be crossed in AMICS, where even if ultimately implanting systems to restore flow would nonetheless be futile.

We previously designed algorithms to implant durable continuous flow LVADs (CF-LVADs) prior to the establishment of the irreversible cascade of multisystem 

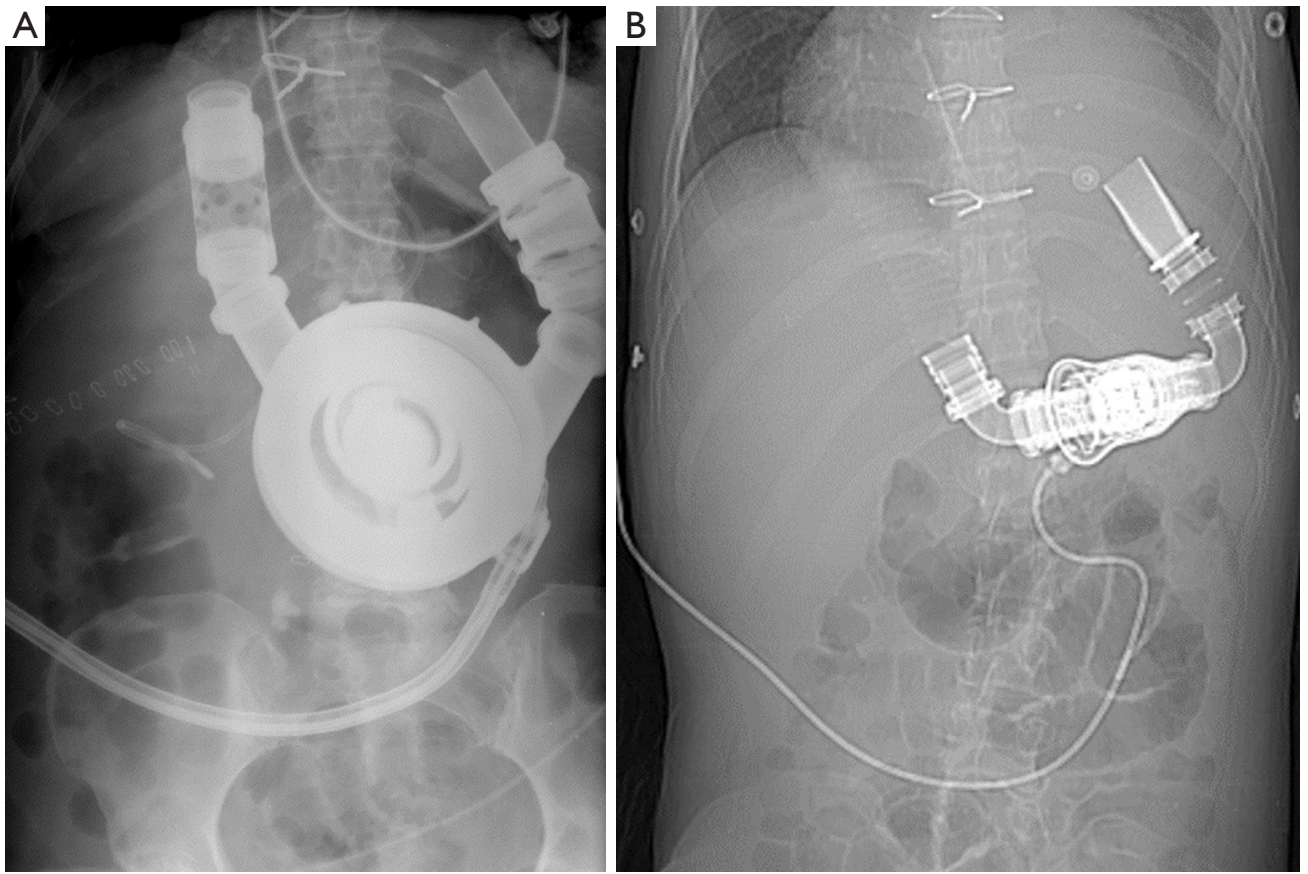

Figure 7 X-ray films of patients with HeartMate XVE LVAD (A) and HeartMate II LVAD (B). LVAD, left ventricular assist device.

organ failure and systematic inflammatory response as shown in Figure 8 (30). This was based on a single institutional non-randomized case series of a select patient population, and in the study, a survival rate of approximately $88 \%$ was reported in patients defined to have refractory cardiogenic shock who underwent primary implantation of CF-LVADs (31). Refractory cardiogenic shock was defined as a continuous shock state, which is clearly distinct from compensated device- or drug-dependent states. The key component of this algorithm is time. Even though this was originally arbitrary, we felt that we could restore flow and simultaneously save the patient if they did not cross the event horizon. Also incumbent in adopting this protocol, it is important to recognize that: (I) myocardial unloading promotes recovery and that recovery was still an ultimate option (II) that these devices had changed over time; lower adverse event profiles, increased durability of these devices, and easier implant methods justified their use. In the design we had placed our focus on saving the patient and not having that immediately dependent on the native heart. In patients implanted in refractory cardiogenic shock, they could recover their native heart function and subsequently undergo cardiac transplantation or remain on the device indefinitely.

This is our current algorithm that we utilize today. As shown in Figure 9, one of the particular aspects of our algorithm is that we utilize hemodynamic parameters as well as clinical signs of organ system perfusion to drive the progression through the decision tree. Within these algorithms, we utilize cardiac power, as we feel that it is one of the more predictive methods of gauging these patients (32). It is also important to develop weaning algorithms for different temporary systems so that weaning is performed in a systematic manner (33).

The development of an acute MCS multidisciplinary team which has the capability to initiate a timely evaluation of the patient's candidacy for the different support strategies and is given the authority to implement these decisions is essential to a programs' success and efficiency. The most important aspect of these algorithms and decision trees is not necessarily in how we do it versus how others do it. The most important element is that a systematic approach is developed across your enterprise encompassing all the phases of care encountered in this population of patients that can be implemented, tracked and refined in a proactive manner to improve their outcomes within individual systems. 


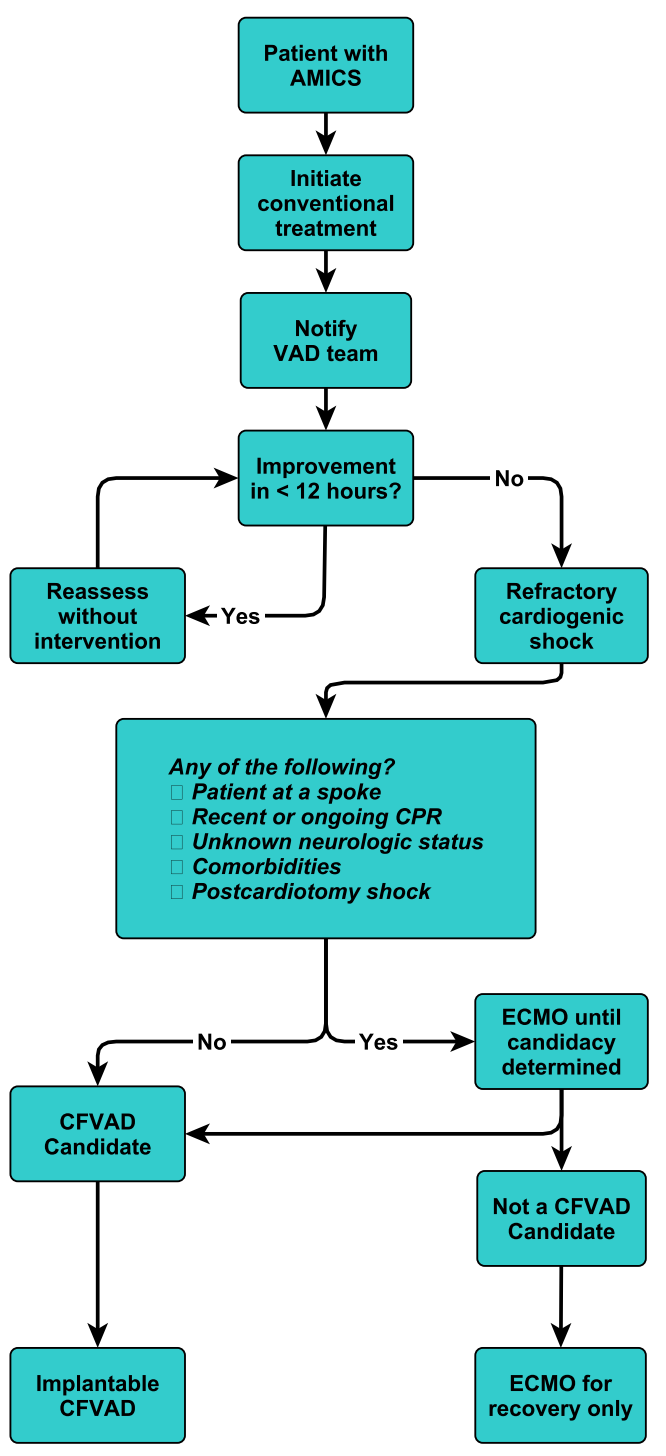

Figure 8 Previous algorithm of MCS strategies for cardiogenic shock, as used by our institution. MCS, mechanical circulatory support; AMICS, acute myocardial infarction with cardiogenic shock; VAD, ventricular assist device; CPR, cardiopulmonary resuscitation; ECMO, extracorporeal membrane oxygenation; CFVAD continuous flow ventricular assist device. Recreated from Tchantchaleishvili et al., 2014 (30).

Going forward in temporary mechanical support, we certainly need systems that are more biocompatible, more durable, can be minimally invasively deployed and minimally invasively decommissioned that have full flow capabilities with low adverse event profiles. The more durable highly biocompatible implantable LVAD systems have continued to develop along the lines of smaller systems with full flow capabilities utilizing less invasive implantation techniques. In time, the acute temporary pumps and chronic systems will to continue to converge and there will be less discrepancy between the systems in utilization.

\section{The new heart allocation system}

The new heart allocation system that is soon to be adopted in the United States is changing the system urgency listing criteria. The system for urgency listing is increasing the urgency status for patients requiring temporary MCS and VA-ECMO. It is unclear what the ramifications of this will be. Due to the strict time limits on device support, there is some concern that individuals may be compelled to remove someone's native heart prior to adequate assessment for the potential of recovery in the fact they may lose a window to transplant an individual at a higher urgency status. The other area of concern is that individuals who have no potential for recovery may actually remain on temporary support systems longer than previously anticipated, in hopes of getting a direct heart transplant and potentially miss a window to be safely transitioned to a long-term support system. For current strategies, the majority of patients on temporary MCS are to utilize these systems to restore perfusion and end organ function to adequately support patients to early native cardiac recovery or to transition to implantable CF-LVAD systems if candidacy criteria are met. The majority of patients supported with temporary MCS systems can be safely transitioned to CFLVADs or total heart replacement. By primarily and more broadly funneling donor hearts to all cardiogenic shock patients on temporary MCS we may inadvertently explant native hearts that ultimately may have recovered, and we may run the risk delaying implant of CF-LVADs, missing a window of survival.

\section{Conclusions}

The use of temporary mechanical support for patients with AMI-CS has substantially increased during the past few decades. Future clinical trials as well as the development and implementation of a systematic evidenced based approach regarding MCS may further guide caregivers in managing patients with cardiogenic shock. 


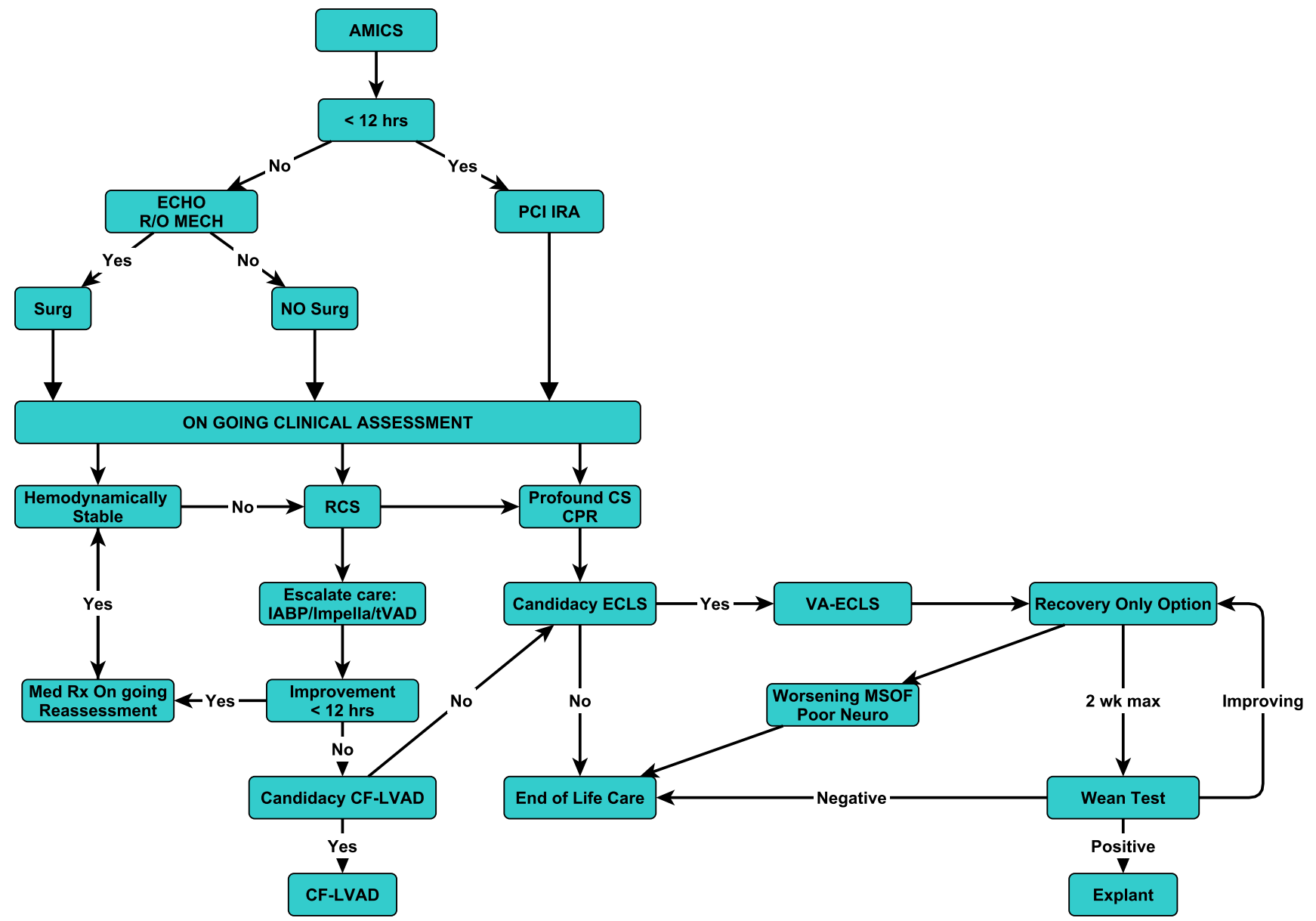

Figure 9 Current algorithm of MCS strategies for cardiogenic shock, as used by our institution. RCS: SBP <90/MAP <60, symptoms of malperfusion (lactic acidosis, oliguria, altered sensorium); or CI <2.2, PCWP >15, MAP <60; or CPO <0.6 W (CO x MAP/451). MCS, mechanical circulatory support; RCS, refractory cardiogenic shock; SBP, systolic blood pressure; MAP, mean arterial pressure; CI, cardiac index; PCWP, pulmonary capillary wedge pressure; CPO, cardiac power output; W, Watt; CO, cardiac output; AMICS, acute myocardial infarction with cardiogenic shock; ECHO, echocardiography; R/O MECH, rule out mechanical heart complications; PCI IRA, percutaneous coronary intervention of the infarct related artery; CS, cardiogenic shock; CPR, cardiopulmonary resuscitation; IABP, intra-aortic balloon pump; tVAD, temporary ventricular assist device; MSOF, multi-system organ failure; VA-ECLS, veno-arterial extracorporeal life support; Med Rx, medical management.

\section{Acknowledgements}

None.

\section{Footnote}

Conflicts of Interest: The authors have no conflicts of interest to declare.

\section{References}

1. Agarwal M, Agrawal S, Garg L, et al. National Trends in the Incidence, Management, and Outcomes of Heart Failure Complications in Patients Hospitalized for STSegment Elevation Myocardial Infarction. Mayo Clin proceedings Innov Qual outcomes 2017;1:26-36.

2. Hochman JS, Buller CE, Sleeper LA, et al. Cardiogenic 
shock complicating acute myocardial infarction--etiologies, management and outcome: a report from the SHOCK Trial Registry. SHould we emergently revascularize Occluded Coronaries for cardiogenic shocK? J Am Coll Cardiol 2000;36:1063-70.

3. Killip T, Kimball JT. Treatment of myocardial infarction in a coronary care unit. Am J Cardiol 1967;20:457-64.

4. Frishman WH, Alpert JS, Killip T. The Coronary (Cardiac) Care Unit at 50 Years: A Major Advance in the Practice of Hospital Medicine. Am J Med 2017;130:1005-6.

5. Hochman JS, Sleeper LA, Webb JG, et al. Early revascularization in acute myocardial infarction complicated by cardiogenic shock. SHOCK Investigators. Should We Emergently Revascularize Occluded Coronaries for Cardiogenic Shock. N Engl J Med 1999;341:625-34.

6. Krumholz HM, Herrin J, Miller LE, et al. Improvements in door-to-balloon time in the United States, 2005 to 2010. Circulation 2011;124:1038-45.

7. Khot UN, Johnson-Wood ML, Geddes JB, et al. Financial impact of reducing door-to-balloon time in ST-elevation myocardial infarction: a single hospital experience. BMC Cardiovasc Disord 2009;9:32.

8. Thiele H, Akin I, Sandri M, et al. PCI Strategies in Patients with Acute Myocardial Infarction and Cardiogenic Shock. N Engl J Med 2017;377:2419-32.

9. Thiele H, Zeymer U, Neumann FJ, et al. Intraaortic Balloon Support for Myocardial Infarction with Cardiogenic Shock. N Engl J Med 2012;367:1287-96.

10. Schumann J, Henrich EC, Strobl H, et al. Inotropic agents and vasodilator strategies for the treatment of cardiogenic shock or low cardiac output syndrome. Cochrane database Syst Rev 2018;1:CD009669.

11. TRIUMPH Investigators, Alexander JH, Reynolds $\mathrm{HR}$, et al. Effect of tilarginine acetate in patients with acute myocardial infarction and cardiogenic shock: the TRIUMPH randomized controlled trial. JAMA 2007;297:1657-66.

12. Menon V. Management of cardiogenic shock complicating acute myocardial infarction. Heart 2002;88:531-7.

13. Ouweneel DM, Engstrom AE, Sjauw KD, et al. Experience from a randomized controlled trial with Impella 2.5 versus IABP in STEMI patients with cardiogenic pre-shock. Int J Cardiol 2016;202:894-6.

14. Ouweneel DM, Eriksen E, Sjauw KD, et al. Percutaneous Mechanical Circulatory Support Versus Intra-Aortic Balloon Pump in Cardiogenic Shock After Acute Myocardial Infarction. J Am Coll Cardiol 2017;69:278-87.
15. Thiele H, Sick P, Boudriot E, et al. Randomized comparison of intra-aortic balloon support with a percutaneous left ventricular assist device in patients with revascularized acute myocardial infarction complicated by cardiogenic shock. Eur Heart J 2005;26:1276-83.

16. Burkhoff D, Cohen H, Brunckhorst C, et al. A randomized multicenter clinical study to evaluate the safety and efficacy of the TandemHeart percutaneous ventricular assist device versus conventional therapy with intraaortic balloon pumping for treatment of cardiogenic shock. Am Heart J 2006;152:469.e1-8.

17. Stretch R, Sauer CM, Yuh DD, et al. National trends in the utilization of short-term mechanical circulatory support: incidence, outcomes, and cost analysis. J Am Coll Cardiol 2014;64:1407-15.

18. Vallabhajosyula S, Arora S, Sakhuja A, et al. Nationwide trends and outcomes of temporary mechanical circulatory support for cardiogenic shock after left ventricular assist device implantation. J Am Coll Cardiol 2018;71:A746.

19. McCarthy FH, McDermott KM, Kini V, et al. Trends in U.S. Extracorporeal Membrane Oxygenation Use and Outcomes: 2002-2012. Semin Thorac Cardiovasc Surg 2015;27:81-8.

20. Tchantchaleishvili V, Hallinan W, Massey HT. Call for Organized Statewide Networks for Management of Acute Myocardial Infarction-Related Cardiogenic Shock. JAMA Surg 2015;150:1025-6.

21. Truesdell AG, Tehrani B, Singh R, et al. 'Combat' Approach to Cardiogenic Shock. Interv Cardiol 2018;13: 81-6.

22. Apolito RA, Greenberg MA, Menegus MA, et al. Impact of the New York State Cardiac Surgery and Percutaneous Coronary Intervention Reporting System on the management of patients with acute myocardial infarction complicated by cardiogenic shock. Am Heart J 2008;155:267-73.

23. Resnic FS, Welt FGP. The Public Health Hazards of Risk Avoidance Associated With Public Reporting of RiskAdjusted Outcomes in Coronary Intervention. J Am Coll Cardiol 2009;53:825-30.

24. Cheng A, Swartz MF, Massey HT. Impella to unload the left ventricle during peripheral extracorporeal membrane oxygenation. ASAIO J 2013;59:533-6.

25. Peek GJ, Killer HM, Reeves R, et al. Early experience with a polymethyl pentene oxygenator for adult extracorporeal life support. ASAIO J 2002;48:480-2.

26. O'Gara PT, Kushner FG, Ascheim DD, et al. 2013 ACCF/ AHA Guideline for the Management of ST-Elevation Myocardial Infarction. J Am Coll Cardiol 2013;61:e78-140. 
27. Jeger RV, Radovanovic D, Hunziker PR, et al. Ten-year trends in the incidence and treatment of cardiogenic shock. Ann Intern Med 2008;149:618-26.

28. Silvestry SC, McGee EC. Less Invasive Techniques for Left Ventricular Assist Device Implantation. Oper Tech Thorac Cardiovasc Surg 2015;20:189-203.

29. Mehta RH, Lopes RD, Ballotta A, et al. Percutaneous coronary intervention or coronary artery bypass surgery for cardiogenic shock and multivessel coronary artery disease? Am Heart J 2010;159:141-7.

30. Tchantchaleishvili V, Schubmehl H, Swartz MF, et al. Evolving strategies in the treatment of acute myocardial infarction-induced cardiogenic shock. Ann Cardiothorac

Cite this article as: Massey HT, Choi JH, Maynes EJ, Tchantchaleishvili V. Temporary support strategies for cardiogenic shock: extracorporeal membrane oxygenation, percutaneous ventricular assist devices and surgically placed extracorporeal ventricular assist devices. Ann Cardiothorac Surg 2019;8(1):32-43. doi: 10.21037/acs.2018.11.05
Surg 2014;3:606-11.

31. Lazar JF, Swartz MF, Schiralli MP, et al. Survival after left ventricular assist device with and without temporary right ventricular support. Ann Thorac Surg 2013;96:2155-9.

32. Fincke R, Hochman JS, Lowe AM, et al. Cardiac power is the strongest hemodynamic correlate of mortality in cardiogenic shock: a report from the SHOCK trial registry. J Am Coll Cardiol 2004;44:340-8.

33. Aissaoui N, Luyt CE, Leprince $\mathrm{P}$, et al. Predictors of successful extracorporeal membrane oxygenation (ECMO) weaning after assistance for refractory cardiogenic shock. Intensive Care Med 2011;37:1738-45. 